\title{
Research on purification of refined carbazole and anthracene by Solvent crystallization
}

\author{
Wenshuang Dai
}

\begin{abstract}
School of Biological medicine and chemical engineering, Liaoning Institute of Science and Technology, Benxi, Liaoning, 117004, China
\end{abstract}

\begin{abstract}
Key Words: crude anthracene; refined carbazole; refined anthracene; solvent crystallization ABSTRACT: Solvent selection is the key of solvent crystallization method. In this paper, the optimal crystallization conditions for xylene and DMF extraction of refined carbazole is studied that the crude carbazole as a raw material, dissolved temperature is $85^{\circ} \mathrm{C}$, crystal-keeping time is $90 \mathrm{~min}$, the solvent ratio is $1.8 \mathrm{~g} / \mathrm{ml}$, the cooling crystallization time is $50 \mathrm{~min}$, the cooling crystallization temperature is $25^{\circ} \mathrm{C}$. The results showed that the process has hardly environmental pollution and the lower cost, the solvent was circularly utilized, the Purity of refined anthracene and carbazole could reach to more than $96 \%$, the total yield of refined anthracene could reach over $67 \%$.
\end{abstract}

\section{Introduction}

Anthracene and carbazole ${ }^{[1]}$ is an important chemical resources in coal tar, more than $90 \%$ anthracene and $100 \%$ of carbazole of the world's is produced from coking byproduct crude anthracene. Anthracene and carbazole are widely used in dye, medicine, pesticide and guide optical electric specialty materials and other fields, with the rapid development of modern fine chemicals and organic synthesis technology, the demand of market of anthracene and carbazole is increase gradually, at the same time, many new applications are being developed.So it is great significance that to explore the technology refining anthracene and carbazole separation from crude anthracene. The method that has been widely adopted refining anthracene and carbazole from crude anthracene include physical separation process, chemical separation process and composite separation process. The physical separation process is divided into solvent crystallization、 distillation、 zone melting method、emulsion membrane method 、emulsion crystallization and zeolite adsorption separation method ${ }^{[4],[5]}$. The chemical separation process is mainly divided into sulfuric acid method and potassium fusion method ${ }^{[8]}$. it is mainly adopted the solvent - distillation coupled process to separate the crude anthracene at foreign and mostly already realize industrialized production, the technology is relatively mature, but higher construction investment and complicated process. Such as the solvent crystallization and distillation process that separate-refined the anthracene and carbazole from anthracene oil- I at France BEFS company, is simple and automated production, large capacity, but larger the equipment investment, the crystallization technical control complex. the reduced pressure distillation-acetophenone washing crystallization process for the company USES Germany ${ }^{[7]}$, can be obtained with purity of $95 \%$ refined anthracene, but has higher dosage of acetophenone as solvent, could be used in the moderate price situation. At present, The solvent method that is generally adopted to refine the crude anthracene at domestic ${ }^{[3]}$, is only suitable to small-scale production, the disadvantage has the longer process flow and consume lots of solvent, higher cost and large pollution. the key to reduce cost, improve product quality and production rate, increase benefits for domestic crude anthracene refined is that choose low toxicity, easy to operate, easy to recycle, good selective solvent ${ }^{[6]}$. 
In this experiment, it is studied that DMF is based the main solvent in the dissolution and crystallization process that be used for the separation and purification of the crude anthracene.

\section{Experiment}

\section{Reagent and Instrument}

In this experiment, the crude anthracene (anthracene $(23.18 \%$ ), carbazole $(23.18 \%)$ as the raw material is from the zhonglian chemical factory. the main solvent that has been selected ,such as ,DMF and dimethyl benzene, chlorobenzene and NMP, DMA etc, are analytical reagent.Main instruments are DF-101s collector heating and constant temperature magnetic agitator, Electrothermal constant temperature water-bath, Water-ring vacuum pump, HDM500tempering temperature electric heating jacket, WRD-1B digital instrument of melting point.

\section{Experimental}

\section{First solvent crystallization}

First, the crude anthracene is joined in the three-necked flask $(250 \mathrm{ml})$. at the same time, the mixed solvent is joined in according to the solid-liquid ratio $1: 1.8$. Second, it is heated to $90^{\circ} \mathrm{C}$ in the thermostatic oil bath pot, stired fully it till be soluble and keeped warm 50min, and then slowly cooling groove to $25^{\circ} \mathrm{C}$ in the constant temperature water bath, crystal growing time 50min. Next step, filter cake by suction filtration is semi-refined anthracene that is dark yellow green and the filtrate of blackish green color. Next step, the solvent will be recycled by vacuum distillation under the temperature of $130^{\circ} \mathrm{C}$ to $140^{\circ} \mathrm{C}$ and the main components of the solid black residue is carbazole and phenanthrene, that is the raw materials for the extraction of carbazole.

\section{Second solvent crystallization}

First, the semi-refined anthracene Will be joined in the three-necked flask that was obtained at the first solvent crystallization, at the same time, the mixed solvent is joined in according to the certain solid-liquid ratio. Second, it is heated to $90^{\circ} \mathrm{C}$ in the thermostatic oil bath pot, stired fully it till be soluble and keeped warm 1hour, and then slowly cooling groove to $25^{\circ} \mathrm{C}$ in the constant temperature water bath, crystal growing time 50min. Next step, filter cake by suction filtration is refined anthracene that is yellowish-green and is dryed in the drying oven. Next step, then the refined anthracene is determinated the purity and calculated the yield according to the YB/T5086-2005 standard method. The technological process is shown in figure 1.

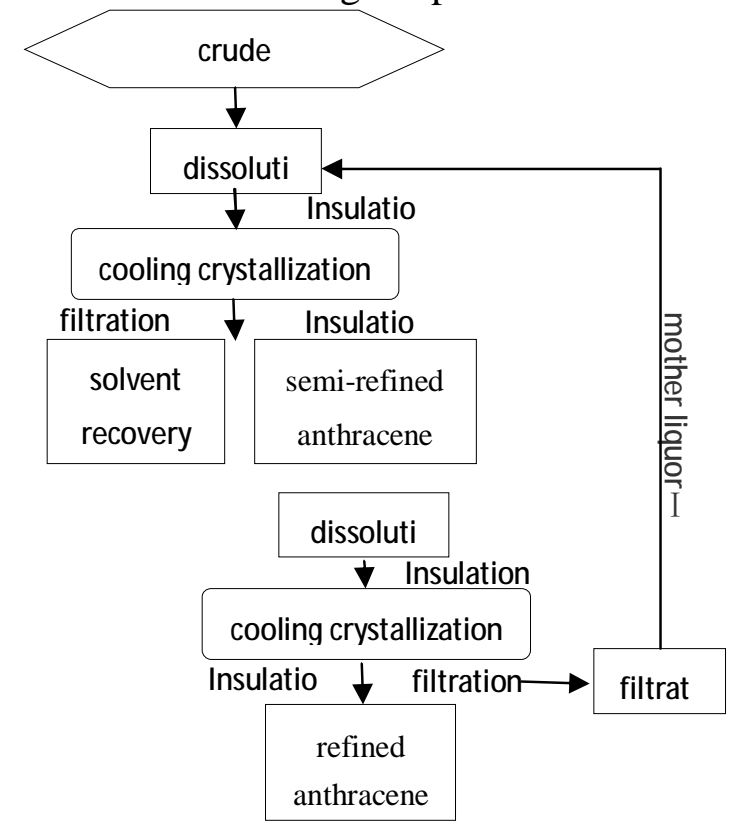

Figure1 Solvent crystallization process flow chart 


\section{Results and discussion}

\section{solvent selection}

In this experiment, the DMF is the main solvent of the mixed solvent preparation to be refined anthracene in which suitable amount of NMP are added. It is studied to the influence of the mixed solvent on the yield and purity of refined anthracene at the dissolution $90^{\circ} \mathrm{C}$ and insulation $50 \mathrm{~min}$, and then slowly cooled to $25^{\circ} \mathrm{C}$ and crystal growing time 50min. By figure 2 and figure 3 , the purity and yield of product changes are flat, there is no special values. when the amount of NMP joined in DMF is less than $10 \%$, the purity of refined-anthracene is below $93 \%$, the yield is $67 \%-69 \%$; When the addition of NMP amount is more than $15 \%$, the purity of refined-anthracene is more than $95 \%$, the yield is 65\%-67\%; When the addition of NMP amount is more than $25 \%$, the purity of refined-anthracene can reach more than $96 \%$, the maximum of yield can reach $68.13 \%$.Considering the purity of industrial refined anthracene with, the amount of NMP is $25 \%$, namely the DMF and NMP compound ratio 3:1.
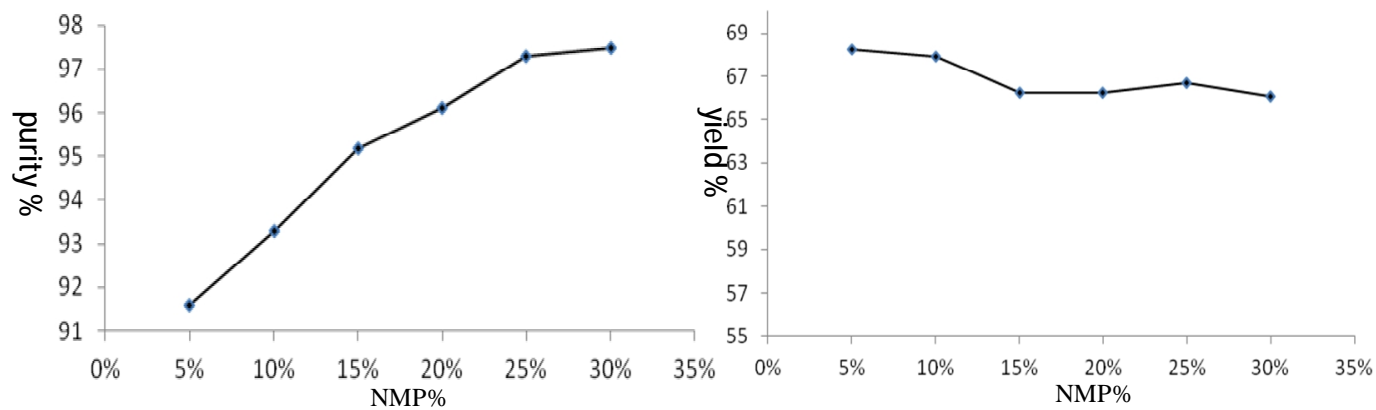

Figure 2 the influence of the mixed solvent on the purity Figure 3 the influence of the mixed solvent on the purity

\section{Solvent dosage}

It can be concluded that when the liquid-solid ratio is less than 1.8 by figures 4 and 5 , the purity of product increased gradually; when liquid-solid ratio is greater than 1.8, increasing the purity of the product is slightly and but the basic stable, its purity is above 95\%; Product yield figure into a parabola, the highest yield when liquid-solid ratio is 1.8. when liquid-solid ratio is greater than 1.8, the yield of product drops rapidly, therefore, the mixed solvent of DMF and NMP the best liquid-solid ratio is 1.8 .

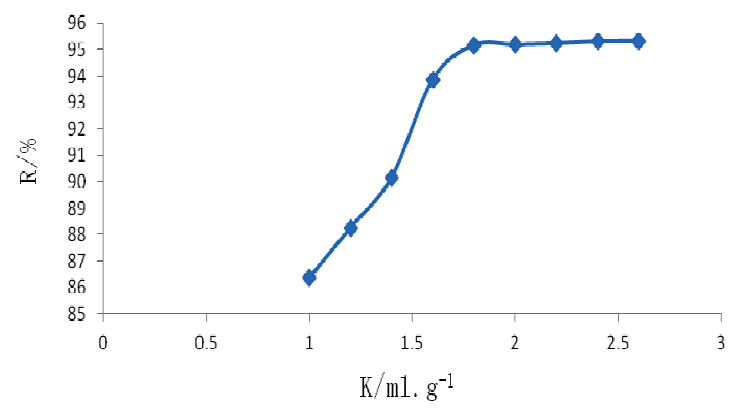

Figure 4 the influence of solvent dosage on the purity

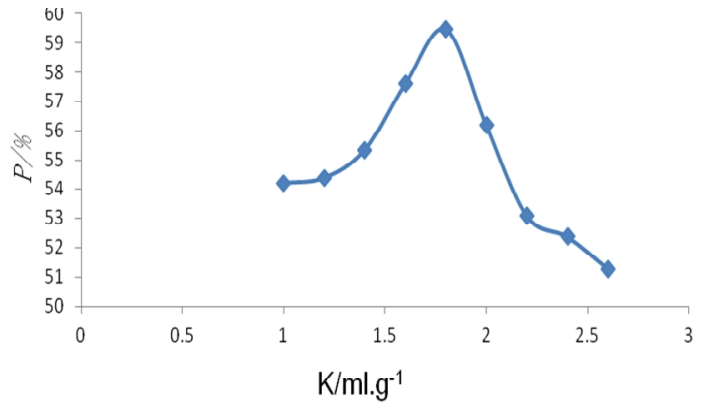

Figure 5 the influence of solvent dosage on the purity

\section{crystallizing point}

With liquid-solid ratio of the mixed solvent is $1.8(\mathrm{ml} / \mathrm{g})$, crystal growing time is 50min, melting temperature is $90^{\circ} \mathrm{C}$, it is studied the influence of different crystallization temperature to the purity of the product. with the increasing temperature, the purity of the anthracene product showes a trend of rise, and the purity of product anthracene with temperature increases with the increase of amplitude is more uniform, but the yield has a downward trend, at the same time ,the content of anthracene in the crude carbazole is larger, it is difficult to the processing of crude carbazole and the refined carbazole. Comprehensive, the cooling temperature is $25^{\circ} \mathrm{C}$. 


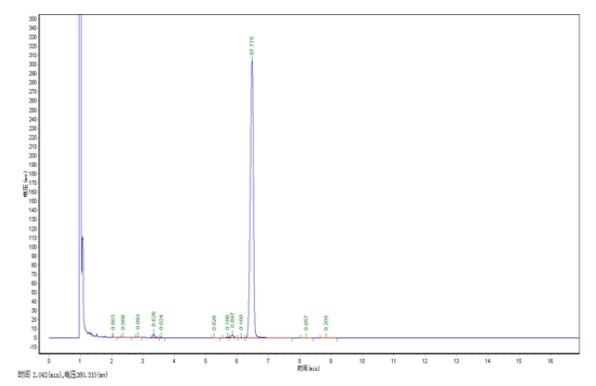

Figure6 GC of refining products by DMF

\section{Conclusion}

The solvent crystallization process is used to be refined anthracene for the crude anthracene as raw materials and DMF as the mixed solvent. the best process conditions: with DMF as solvent, NMP as cooperate with solvent, best compound ratio 3:1, best liquid-solid ratio $1.8 \mathrm{ml} / \mathrm{g}$ and the dissolution $90^{\circ} \mathrm{C}$ and holding time $50 \mathrm{~min}$, the cooling crystallization temperature $25^{\circ} \mathrm{C}$ and holding time $50 \mathrm{~min}$. This method is simple in process and easy to recycle solvent, the low cost.the purity of refined anthracene was up to $96 \%$ and the yield is $67 \%$.

\section{Reference}

[1] Rhua Xiao, coal tar chemical technology. [M].beijing: Metallurgical Industry Press, 2009.2. [2]Zhengbo Zhao. The choice of solvent to process of separation of crude anthracene [D]. taiyuan: Taiyuan University of Technology Press, 2003.

[3]He Meng. Separation and purification method of Anthracene and carbazole [J]. Shanxi chemical industry, 2003(4):4-7。

[4] Jianmin Yang. Progress in the production technology of refined Anthracene [J]. Coal chemical industry, 2004(4): 13-15.

[5]Cuiping Ye. study of refining high pure anthracene carbazole at solvent crystallization [J]. Coal conversion,2013.1: 83-87.

[6]Zhengzai Chen. Separation and purification of main components in crude anthracene [J]. Coal conversion, 2013,36 (3): 68-71.

[7]Qiusheng Xie. Study on production of carbazole extraction method [J]. Fuel and chemical, 2003, 34(3): 153-154.

[8]Aihua Liu. Study on Extraction of refined anthracene from crude anthracene [J]. Taiyuan University of Technology Press 2007(3): 233-235. 